\section{COPD: Pulmonale Hypertonie im CT diagnostizierbar?}

Mohamed Hoesein FA et al. Accuracy of CT Pulmonary Artery Diameter for Pulmonary Hypertension in End-Stage COPD. Lung 2016; 194: $813-819$

Die Computertomografie (CT) erlaubt bei fortgeschrittener COPD nur bedingt valide Aussagen zum Grad der begleitenden pulmonal-arteriellen Hypertonie (PAH).

Die Diagnose und Klassifizierung der PAH bei fortgeschrittener COPD ist ein wichtiges Transplantationskriterium. Als Standard gilt hier die invasive Messung mittels Rechtsherzkatheter. Bei dieser Fragestellung konkurrieren auch bildgebende Techniken (CT). Hierbei wird entweder der Durchmesser der Pulmonalarterie (PA) oder das Verhältnis PA zur aszendierenden Aorta als PAH-Kriterium genutzt. Über die Aussagekraft beider Kenngrößen besteht noch Unklarheit.

Im Rahmen ihrer retrospektiven Analyse werteten die Autoren die Daten von 92 Patienten mit fortgeschrittener COPD oder alpha-1-Antitrypsin-MangelEmphysem aus. Sie nutzten hierzu das Transplantationsregister der Universität Utrecht (2004-2014). Bei allen Betroffenen war eine Transplantation geplant. Neben der bildgebenden Diagnostik (CT) lag auch ein invasiver Befund (Rechtsherzkatheter) vor. Die Forscher prüften, welchen Vorhersagewert die Parameter „Durchmesser PA“ und „Verhältnis PA/ Aorta“ in Bezug auf die Inzidenz der PAH aufwiesen. Als aussagekräftigen Schwellenwert definierten sie einen, durch Rechtsherzkatheter gemessenen, mittleren pulmonal-arteriellen Druck > 25 $\mathrm{mmHg}$.

\section{Ergebnisse}

Die Patienten im Alter von ca. 55 Jahren wiesen eine durchschnittliche AusgangsFEV1 von 23 \% des Erwartungswertes auf. Ihre Nikotinbelastung schwankte zwischen 26 und 31 Packungsjahren. Bei $33 \%$ der Patienten ließ sich die PAH in- vasiv bestätigen. In dieser Gruppe betrug der mittlere pulmonal-arterielle Druck $30 \mathrm{mmHg}$. In der Gruppe ohne Nachweis lag der Wert bei $19 \mathrm{mmHg}$. Bei einem als Cut-Off definierten PA-Durchmesser von $25 \mathrm{~mm}$ erreichte die Sensitivität 0,9 , bei einer Spezifität von 0,6. Der positive Vorhersagewert (PPV) lag demnach bei 0,52, der negative Vorhersagewert (NPV) bei 0,92. Ein Schwellenwert von $31 \mathrm{~mm}$ dagegen erhöhte die Spezifität $(0,95)$ auf Kosten der Sensitivität $(0,37)$. Die Vorhersageparameter betrugen: PPV 0,79 bzw. NPV 0,76. Ein ähnliches Bild zeigte sich auch beim Verhältnis PA/ Aorta. Sensitivität und Spezifität dieses Parameters variierten ebenfalls stark abhängig vom gewählten Cut-Off. Bei einem PA/Aorta-Schwellenwert $\geq 0,9$ betrug die Sensitivität 0,7, bei einer Spezifität von 0,63 . Ein hoher Schwellenwert $(\geq 1,1)$ verminderte die Sensitivität drastisch $(0,17)$, verbesserte dafür aber die Spezifität $(0,95)$. Der positive Vorhersagewert lag dann bei 0,63 , der negative bei 0,70 .

\section{FAZIT}

Die Verdachtsdiagnose pulmonalarterielle Hypertonie bei der fortgeschrittenen COPD ist auch auf der Basis von CT-Parametern möglich. Allerdings muss hier die hohe Divergenz zwischen Spezifität und Sensitivität beachtet werden. Unter diesem Gesichtspunkt bewerten die Autoren die morphologisch-radiologische Diagnostik der PAH bei COPD als nur bedingt valide.

Dr. med. Horst Gross, Berlin 